\title{
Evidence-Based PET for Endocrine Tumours and Disorders
}

\author{
Alexander Stephan Kroiss and Giorgio Treglia
}

\subsection{Introduction}

Endocrine tumours have a wide range of clinical presentations and can be found anywhere from the neck to the pelvis. Diagnostic imaging is crucial to predict the exact tumour extent, foremost in metastatic disease. Anatomical imaging as computed tomography (CT) and magnetic resonance imaging (MRI) serves as the first-line modality in the locoregional staging of these tumours. Compared with anatomical imaging, PET shows both high sensitivity and specificity. Several meta-analyses have described the diagnostic performance of positron emission tomography (PET) and hybrid imaging (PET/CT) in endocrine tumours and disorders.

\footnotetext{
A. S. Kroiss $(\square)$

Department of Nuclear Medicine, Medical University Innsbruck, Innsbruck, Austria

e-mail: alexander.kroiss@i-med.ac.at

\section{G. Treglia}

Clinic of Nuclear Medicine and PET/CT Center, Imaging Institute of Southern Switzerland, Ente Ospedaliero Cantonale,

Bellinzona and Lugano, Switzerland

Health Technology Assessment Unit, Ente Ospedaliero Cantonale, Bellinzona, Switzerland

Department of Nuclear Medicine and Molecular Imaging, Lausanne University Hospital and University of Lausanne, Lausanne, Switzerland e-mail: giorgio.treglia@eoc.ch
}

\subsection{Adrenal Tumours and Paragangliomas}

\subsubsection{Characterization of Adrenal Masses}

Dinnes and colleagues reviewed the evidence on the accuracy of imaging tests for differentiating malignant from benign adrenal masses. They concluded that CT density $>10$ Hounsfield Unit (HU) offers high sensitivity for detection of adrenal malignancy in participants with no prior indication for adrenal imaging. With respect to a limited database and heterogeneity and low quality of included studies for meta-analysis, the authors concluded that there is insufficient evidence for the diagnostic value of individual imaging tests in distinguishing benign from malignant adrenal masses [1].

Kim and colleagues explored the role of the diagnostic accuracy of ${ }^{18} \mathrm{~F}$-FDG PET or PET/CT for characterization of adrenal lesions [2]. The pooled sensitivity for ${ }^{18} \mathrm{~F}-\mathrm{FDG}$ PET or PET/CT was $91 \%$ (95\% confidence interval $(95 \% \mathrm{CI})$ : $88-94 \%$ ) and the pooled specificity was $91 \%$ (95\%CI: 87-93\%). Although, at present, the literature regarding the use of ${ }^{18} \mathrm{~F}-\mathrm{FDG}$ PET or PET/CT for the characterization of adrenal masses remains limited, ${ }^{18} \mathrm{~F}-\mathrm{FDG}$ PET or PET/ CT demonstrated good sensitivity and specificity for the characterization of adrenal masses. 


\subsubsection{Paragangliomas}

Rufini and co-authors compared the diagnostic performance of metaiodobenzylguanidine (MIBG scintigraphy) and PET with different radiopharmaceuticals in patients with paraganglioma (PGL). The authors concluded that the diagnostic performance of PET with different radiopharmaceuticals is clearly superior to that of MIBG scintigraphy in patients with PGL, mainly for familial, extra-adrenal and metastatic diseases [3].

A review article by Treglia and co-authors investigated the diagnostic performance of ${ }^{18} \mathrm{~F}$-DOPA PET in patients with paraganglioma (PGL). The pooled sensitivity of ${ }^{18} \mathrm{~F}-\mathrm{DOPA}$ PET and PET/CT in detecting PGL was $91 \%$ (95\%CI: $87-94 \%$ ) on a per-patient-based analysis and 79\% (95\% CI 76-81\%) on a per-lesionbased analysis. The pooled specificity of ${ }^{18} \mathrm{~F}$-DOPA PET and PET/CT in detecting PGL was 95\% (95\%CI: 86-99\%) on a per-patientbased analysis and 95\% (95\%CI: 84-99\%) on a per-lesion-based analysis. The area under the receiver operating characteristic (ROC) curve was 0.95 on a per-patient- and 0.94 on a perlesion-based analysis. The authors described the possible risk of false-negative ${ }^{18} \mathrm{~F}$-DOPA PET results in metastatic PGL, besides the fact that succinate dehydrogenase subunit B (SDHB) gene mutations could influence the diagnostic performance of ${ }^{18} \mathrm{~F}-\mathrm{DOPA}$ PET or PET/CT [4].

Kan and colleagues performed a meta-analysis on the localization of metastatic pheochromocytoma (PHEO) and PGL with germline mutations, comparing ${ }^{68} \mathrm{Ga}$-somatostatin analogues and ${ }^{18} \mathrm{~F}$ FDG PET/CT. The pooled sensitivity of ${ }^{68} \mathrm{Ga}$ peptides and ${ }^{18} \mathrm{~F}-\mathrm{FDG}$ PET were $95 \%$ (95\%CI:92-97) and 85\% (95\%CI:78-91\%), respectively. The pooled specificity of ${ }^{68} \mathrm{Ga}$ peptides and ${ }^{18} \mathrm{~F}-\mathrm{FDG}$ PET were $87 \%$ (95\%CI: 63-96\%) and 55\% (95\%CI:37-73\%), respectively. The authors concluded that ${ }^{68} \mathrm{Ga}$-somatostatin analogues PET/CT demonstrated good performance in the localization of metastatic PGL, especially those with germline mutations, compared to ${ }^{18} \mathrm{~F}-\mathrm{FDG}$ PET/CT [5].

Han and colleagues performed a systematic review and meta-analysis on the performance of ${ }^{68} \mathrm{Ga}$-somatostatin analogues PET in the detection of PGL. The pooled detection rate was $93 \%$ (95\%CI: 91-95\%), which was significantly higher than that of ${ }^{18} \mathrm{~F}-\mathrm{DOPA}$ PET $(80 \%$; $95 \% \mathrm{CI}$ : 69-88\%), ${ }^{18} \mathrm{~F}-\mathrm{FDG}$ PET (74\%; 95\%CI: 46-91\%) and MIBG scintigraphy (38\%; 95\%CI: $20-59 \%)$. A greater prevalence of head and neck PGL was associated with higher detection rates of ${ }^{68} \mathrm{Ga}$-somatostatin analogues PET. The authors suggest the use of ${ }^{68} \mathrm{Ga}$-somatostatin analogues PET as a first-line imaging modality for the primary staging or restaging of PGL with unknown genetic status [6].

\subsection{Neuroblastoma}

Bleeker and colleagues described the role of MIBG scintigraphy and ${ }^{18} \mathrm{~F}-\mathrm{FDG}$ PET for diagnosing neuroblastoma (NB). In one study, the sensitivity of ${ }^{18} \mathrm{~F}-\mathrm{FDG} \mathrm{PET} / \mathrm{CT}$ compared to MIBG scintigraphy was $100 \%$ and $92 \%$, respectively. Specificity could not be calculated for both modalities. The diagnostic accuracy of ${ }^{18} \mathrm{~F}-\mathrm{FDG}$ PET/CT imaging in case of a negative ${ }^{123} \mathrm{I}-\mathrm{MIBG}$ scintigraphy could not be calculated because of very limited data. It has to be mentioned that in about $10 \%$ of the patients with histologically proven NB the tumour does not accumulate ${ }^{123} \mathrm{I}-\mathrm{MIBG}$ which underlines the importance of additional functional/anatomical imaging (e.g. ${ }^{18}$ F-FDG PET/CT) [7].

A review article by Xia and co-authors demonstrated a summary sensitivity for MIBG scintigraphy and ${ }^{18}$ F-FDG PET(/CT) of $79 \%$ and $89 \%$, respectively. The summary specificity for MIBG scintigraphy and ${ }^{18} \mathrm{~F}-\mathrm{FDG}$ PET(/CT) was $84 \%$ and $71 \%$, respectively. The authors concluded that ${ }^{18} \mathrm{~F}$-FDG PET(/CT) showed higher per-lesion accuracy than MIBG scintigraphy and might be the preferred modality for the staging of NB [8]. 


\subsection{Merkel Cell Carcinoma}

Treglia and co-authors investigated the diagnostic performance of ${ }^{18} \mathrm{~F}-\mathrm{FDG}$ PET and PET/CT in patients with Merkel cell carcinoma (MCC). The meta-analysis provided the following pooled results on a per-examination-based analysis: sensitivity was 90\% (95\%CI: 80-96\%) and specificity was 98\% (95\%CI: 90-100\%). The area under the summary ROC curve was 0.96 . No significant statistical heterogeneity between the studies was found. The authors concluded that ${ }^{18} \mathrm{~F}$-FDG PET or PET/CT demonstrated high sensitivity and specificity, being accurate methods in the detection of MCC taking into account that literature in MCC remains limited [9].

\subsection{Gastroenteropancreatic and Pulmonary Neuroendocrine Tumours}

Singh and co-authors evaluated the diagnostic performance of ${ }^{68} \mathrm{Ga}$-somatostatin analogues PET or PET/CT on neuroendocrine tumours (NETs). For the initial diagnosis of NETs, ${ }^{68} \mathrm{Ga}$-somatostatin analogues PET or PET/CT had a pooled sensitivity of $91 \%$ (95\%CI: $85-94 \%$ ) and a pooled specificity of $94 \%(95 \% \mathrm{CI}$ : 86-98\%). In the setting of staging and restaging, the sensitivity of ${ }^{68} \mathrm{Ga}$-somatostatin analogues PET or PET/CT for detecting primary and/or metastatic lesions ranged from 78.3 to $100 \%$, whereas specificity ranged from 83 to $100 \%$. Change in management occurred in $45 \%$ (95\% CI: $36-55 \%)$ of the cases, with majority of the changes involving surgical planning and patient selection for peptide receptor radionuclide therapy [10].

This is in line with a systematic review by Barrio and colleagues who investigated the impact of ${ }^{68} \mathrm{Ga}$-somatostatin analogues PET/CT in patients with NETs. A change of management occurred in $44 \%$ of cases after ${ }^{68} \mathrm{Ga}$-somatostatin analogues PET/CT (range: 16-71\%). In some studies, ${ }^{68} \mathrm{Ga}$-somatostatin analogues PET/CT was performed after conventional scintigraphy ( ${ }^{111}$ In-Octreotide). In this subgroup, additional information led to a change in management in $39 \%$ of cases (range: $16-71 \%$ ). The authors concluded that the management was changed in more than one-third of patients undergoing ${ }^{68} \mathrm{Ga}$-somatostatin analogues PET/CT even when performed after an ${ }^{111} \mathrm{In}$-Octreotide scintigraphy [11].

In this line, another meta-analysis was published by Deppen and co-authors who compared conventional ${ }^{111}$ In-Octreotide imaging with ${ }^{68} \mathrm{Ga}$-DOTATATE PET/CT in pulmonary and gastroenteropancreatic NETs, with estimated pooled sensitivity of $90.9 \%$ (95\%CI: $81.4-96.4 \%)$ and pooled specificity of $90.6 \%$ (95\%CI: 77.8$96.1 \%$ ) for ${ }^{68} \mathrm{Ga}$-DOTATATE PET/CT [12].

The high diagnostic performance of ${ }^{68} \mathrm{Ga}$-somatostatin analogues PET or PET/CT for thoracic and gastroenteropancreatic NETs was showed by the meta-analysis of Treglia et al. reporting a pooled sensitivity and specificity of 93\% (95\% CI: 91-95\%) and 91\% (95\% CI: 82-97\%), respectively. ${ }^{68} \mathrm{Ga}$-somatostatin analogues PET/CT should be considered as first-line diagnostic imaging method for these tumours [13].

An updated meta-analysis on this regard reported a pooled sensitivity of $93 \%$ (95\% CI: $91-94 \%$ ) and a pooled specificity of $96 \%$ (95\%CI: 95-98\%) for ${ }^{68} \mathrm{Ga}$-somatostatin analogues PET or PET/CT. The area under the summary ROC curve was 0.98 , confirming the good diagnostic performance of ${ }^{68} \mathrm{Ga}$-somatostatin analogues PET or PET/CT compared to diagnostic CT and conventional scintigraphy (e.g. ${ }^{111}$ In-Octreotide) [14].

An evidence-based article compared ${ }^{68} \mathrm{Ga}$-DOTATOC and ${ }^{68} \mathrm{Ga}$-DOTATATE PET in NETs, reporting no statistically significant differences of diagnostic performance among these imaging methods on a per-patient-based analysis [15].

\subsection{Congenital Hyperinsulinism}

Paediatric patients with congenital hyperinsulinism (CHI) due to pancreatic disease can be evaluated by PET or PET/CT, in particular by using 
${ }^{18}$ F-DOPA. A systematic review and metaanalysis by Blomberg and co-authors aimed to quantify the diagnostic performance of pancreatic venous sampling (PVS), selective pancreatic arterial calcium stimulation with hepatic venous sampling (ASVS) and ${ }^{18} \mathrm{~F}$-DOPA PET in diagnosing and localizing focal form of $\mathrm{CHI}$. ${ }^{18} \mathrm{~F}$-DOPA PET was superior in distinguishing focal from diffuse CHI compared to PVS and ASVS. Furthermore, it localized focal CHI in the pancreas more accurately than PVS and ASVS (pooled accuracy: $82 \%$ vs. $76 \%$ and $64 \%$, respectively) [16].

Yang and colleagues performed a metaanalysis of published data on the diagnostic role of ${ }^{18} \mathrm{~F}$-DOPA PET in patients with CHI. The pooled sensitivity of ${ }^{18} \mathrm{~F}$-DOPA PET and PET/CT in detecting $\mathrm{CHI}$ was $88 \%$. The pooled specificity of ${ }^{18} \mathrm{~F}$-DOPA PET and PET/CT in demonstrating $\mathrm{CHI}$ was $79 \%$. The area under the ROC curve was 0.92 . The authors concluded that ${ }^{18} \mathrm{~F}$-DOPA PET or PET/CT demonstrated high sensitivity and specificity in patients with CHI [17].

These findings are in line with another metaanalysis by Treglia and co-authors: the pooled sensitivity and specificity of ${ }^{18} \mathrm{~F}$-DOPA PET or PET/CT in differentiating between focal and diffuse $\mathrm{CHI}$ were $89 \%$ (95\% CI: $81-95 \%$ ) and $98 \%$ (95\%CI: 89-100\%), respectively. The area under the ROC curve was 0.95 . The pooled accuracy of these functional imaging methods in localizing focal CHI was $80 \%$ (95\%CI: 71-88\%). Although possible sources of false-negative results for focal CHI should be kept in mind, the authors concluded that ${ }^{18} \mathrm{~F}-\mathrm{DOPA}$ PET or PET/CT are accurate methods for localizing focal CHI [18].

\subsection{Thyroid Diseases}

\subsubsection{Thyroid Incidentalomas}

Nayan and colleagues evaluated through a systematic review and meta-analysis the malignancy rates of thyroid incidentalomas identified in adults by ${ }^{18} \mathrm{FDG}$ PET/CT. The pooled proportion of malignancy was $19.8 \%$ (95\%CI: $15.3-24.7 \%$ ) with most of cases being papillary thyroid cancer.
The authors stated that thyroid incidentalomas identified through ${ }^{18} \mathrm{FDG}$ PET require thorough investigation [19].

In this context, a review article by $\mathrm{Qu}$ and coauthors was focused on focal thyroid incidentalomas (FTI) identified on ${ }^{18} \mathrm{~F}$-FDG PET or PET/ CT. A meta-analysis was performed to investigate whether the maximum standardized uptake value (SUVmax) could discriminate between benign and malignant FTI and to explore the cutoff value of SUVmax for the diagnosis of malignancy. The results of this article indicated that there was no statistically significant difference in the size between benign and malignant FTI, while a significantly higher SUVmax was observed in the malignant group. The authors concluded that a higher SUVmax in FTI was associated with a higher risk of thyroid malignancy, especially at a threshold of 3.3 or more [20].

Treglia and co-authors described the prevalence and malignancy risk of FTI detected by ${ }^{18} \mathrm{~F}-$ FDG PET or PET/CT. Pooled prevalence of FTI was $1.92 \%$ (95\%CI: $1.87-1.99 \%)$. Considering FTI which underwent histopathology evaluation, the pooled risk of malignancy was $36.2 \%$ (95\%CI: 33.8-38.6\%), without significant differences among various geographic areas. The authors concluded that FTI are observed in about $2 \%$ of ${ }^{18} \mathrm{~F}-\mathrm{FDG}$-PET or PET/CT and they should be further investigated due to a significant risk of malignancy [21].

\subsubsection{Indeterminate Thyroid Nodules}

A meta-analysis by Wang and colleagues evaluated the diagnostic accuracy of ${ }^{18} \mathrm{~F}-\mathrm{FDG}$ PET or $\mathrm{PET} / \mathrm{CT}$ in discriminating between malignant and benign lesions in thyroid nodules with indeterminate fine needle aspiration biopsy (FNAB). The prevalence of malignant lesions in these patients was $26.2 \%$ (ranging from 19.6 to $40 \%$ ). The pooled sensitivity and specificity of ${ }^{18} \mathrm{~F}-\mathrm{FDG}$ PET or PET/CT for the detection of cancer were $89.0 \%$ (95\% CI: $79.0-95 \%$ ) and 55\% (95\% CI: 48-62\%), respectively. Although SUVmax was 
higher in malignant lesions, there was still a great overlap with benign lesions. In conclusion, ${ }^{18} \mathrm{~F}-$ FDG PET or PET/CT showed a high sensitivity in detecting thyroid cancers in patients with indeterminate FNAB results [22].

\subsubsection{Recurrence of Differentiated Thyroid Cancer}

A meta-analysis by Haslerud and co-authors described the role of ${ }^{18} \mathrm{~F}$-FDG PET in recurrent differentiated thyroid cancer (DTC) after total thyroidectomy and radioiodine ablative therapy. Pooled sensitivity and specificity of this method in detecting recurrent DTC were $79.4 \%$ (95\%CI: 73.9-84.1\%) and 79.4\% (95\%CI: 71.2-85.4\%), respectively, with an area under the ROC curve of 0.858. The authors concluded that this method can be useful for detecting recurrent DTC in patients having undergone radioiodine ablative therapy [23].

A meta-analysis by Caetano and co-authors aimed to evaluate the accuracy of ${ }^{18} \mathrm{~F}$-FDG PET and PET/CT for detecting recurrence of DTC, not identified by ${ }^{131} \mathrm{I}$ whole-body scintigraphy (I-WBS). The combined sensitivity, specificity and accuracy for ${ }^{18} \mathrm{~F}$-FDG PET were $84 \%, 84 \%$ and $91 \%$, respectively; for ${ }^{18} \mathrm{~F}-\mathrm{FDG}$ PET/CT, the combined sensitivity, specificity and accuracy were $93 \%, 81 \%$ and $93 \%$ respectively [24].

Another meta-analysis by Schütz and coauthors about the use of ${ }^{18} \mathrm{~F}$-FDG PET and PET/ CT for detecting recurrent DTC demonstrated that ${ }^{18} \mathrm{~F}-\mathrm{FDG}$ PET and PET/CT showed higher sensitivity (89.7\% for PET and $94.3 \%$ for PET/ CT) compared with conventional imaging $(65.4 \%)$ and comparable results for specificity [25].

Kim and colleagues investigated the diagnostic accuracy of ${ }^{18}$ F-FDG PET/CT for the detection of recurrent and/or metastatic diseases in DTC patients with progressively and/or persistently elevated thyroglobulin antibodies $(\mathrm{TgAb})$ levels and negative I-WBS through a systematic review and meta-analysis. The pooled sensitivity for ${ }^{18} \mathrm{~F}-\mathrm{FDG}$ PET or PET/CT was $84 \%$ (95\%CI: $77-89 \%)$, the pooled specificity $78 \%$ (95\%CI:
$67-86 \%)$. The area under the ROC curve was 0.88 (95\% CI: 0.85-0.90). The authors concluded that ${ }^{18} \mathrm{~F}-\mathrm{FDG}$ PET or PET/CT demonstrated moderate sensitivity and specificity for the detection of recurrent and/or metastatic diseases in DTC patients with progressively and/or persistently elevated $\mathrm{TgAb}$ levels and negative I-WBS [26].

A meta-analysis by Santhanam and co-authors investigated the accuracy of ${ }^{18} \mathrm{~F}-\mathrm{FDG}$ PET/CT in the detection of residual disease in patients with BRAF $^{\mathrm{V} 600 \mathrm{E}}$ mutated thyroid cancer. The authors demonstrated that presence of $\mathrm{BRAF}^{\mathrm{V} 600 \mathrm{E}}$ mutation in DTC confers a higher likelihood of ${ }^{18} \mathrm{~F}$ FDG avidity and is associated with higher SUVmax values compared to $\mathrm{BRAF}^{\mathrm{V} 600 \mathrm{E}_{-}}$ mutation negative status [27].

The role of ${ }^{124} \mathrm{I}-\mathrm{PET} / \mathrm{CT}$ in detecting lesions of DTC amenable to ${ }^{131}$ I-therapy was recently described. The pooled sensitivity of ${ }^{124} \mathrm{I}-\mathrm{PET} / \mathrm{CT}$ in detecting DTC lesions amenable to ${ }^{131} \mathrm{I}$-therapy was $94.2 \%$ (95\% CI: 91.3-96.4\%), and the pooled specificity was $49.0 \%$ (95\% CI: 34.8-63.4\%). The authors concluded that ${ }^{124}$ I-PET/CT is a sensitive tool to diagnose radioiodine-avid DTC lesions, but also detects some new lesions that are not visualized on the post-treatment I-WBS [28].

\subsubsection{Recurrence of Medullary Thyroid Cancer}

Treglia and co-authors described the role of ${ }^{18} \mathrm{~F}$ FDG PET or PET/CT in patients with suspected recurrent medullary thyroid cancer (MTC). A sub-analysis considering PET device used, serum calcitonin, carcino-embryonic antigen (CEA), calcitonin doubling time (CTDT) and CEA doubling time (CEADT) values was also performed. Detection rate (DR) of ${ }^{18} \mathrm{~F}$-FDG PET or PET/CT in suspected recurrent MTC on a per-patientbased analysis was 59\% (95\%CI: 54-63\%). DR increased in patients with serum calcitonin $\geq 1000 \mathrm{ng} / \mathrm{L}$ (75\%), CEA $\geq 5 \mathrm{ng} / \mathrm{mL}$ (69\%), CTDT $<12$ months (76\%) and CEADT $<24$ months $(91 \%)$. The authors reported that about $40 \%$ of suspected recurrent MTC remain usually unidentified by ${ }^{18} \mathrm{~F}-\mathrm{FDG}$ PET or PET/ 
CT. However, ${ }^{18}$ F-FDG PET and PET/CT could modify the patient management in a certain number of recurrent MTC because these methods are often performed after negative conventional imaging studies [29].

In another meta-analysis evaluating the diagnostic performance of ${ }^{18} \mathrm{~F}-\mathrm{FDG}$ and PET/CT for detection of recurrent or metastatic MTC, the pooled sensitivities of ${ }^{18} \mathrm{~F}-\mathrm{FDG}-\mathrm{PET}$ and PET/ CT were 68\% (95\%CI: $64-72 \%)$ and $69 \%$ (95\%CI: 64-74\%), respectively [30].

Other PET radiotracers beyond ${ }^{18} \mathrm{~F}-\mathrm{FDG}$ were evaluated for detecting recurrent MTC. In a metaanalysis evaluating the DR of ${ }^{18} \mathrm{~F}$-DOPA PET or PET/CT for recurrent MTC, the DR of ${ }^{18} \mathrm{~F}-\mathrm{DOPA}$ PET and PET/CT on a per-patient- and a perlesion-based analysis was $66 \%$ and $71 \%$, respectively. The DR significantly increased in patients with serum calcitonin $\geq 1000 \mathrm{ng} / \mathrm{L}(86 \%)$ and CTDT $<24$ months $(86 \%)$. Therefore, ${ }^{18} \mathrm{~F}$-DOPA PET/CT may be a very useful functional imaging method in detecting recurrent MTC [31].

Another meta-analysis assessed the DR of ${ }^{68} \mathrm{Ga}$-somatostatin analogues PET or PET/CT in patients with recurrent MTC. The DR on a perpatient-based analysis was $63.5 \% \quad(95 \% \mathrm{CI}$ : 49-77\%). DR of ${ }^{68} \mathrm{Ga}$-somatostatin analogues PET or PET/CT increased in patients with higher serum calcitonin levels (83\% for calcitonin $>500 \mathrm{ng} / \mathrm{L}$ ). The authors concluded that the diagnostic performance of ${ }^{68} \mathrm{Ga}$-somatostatin analogues PET or PET/CT in recurrent MTC was lower compared to that of the same imaging method in the majority of NETs [32].

\subsection{Parathyroid Diseases}

Different PET tracers may be used to detect hyperfunctioning parathyroid glands in patients with hyperparathyroidism (HPT), including ${ }^{11} \mathrm{C}$-methionine $\left({ }^{11} \mathrm{C}-\mathrm{MET}\right)$ and radiolabelled choline. ${ }^{11} \mathrm{C}$-MET PET has an overall good sensitivity $(69 \%)$ and positive predictive value $(98 \%)$ in detecting hyperfunctioning parathyroid glands in patients with HPT and it may be considered a reliable second-line imaging method to enable minimally invasive parathyroidectomy [33].
Yuan and co-authors published a metaanalysis on the diagnostic value of ${ }^{11} \mathrm{C}$-MET PET in detecting hyperfunctioning parathyroid glands in patients with HPT and negative ${ }^{99} \mathrm{~m}$ Tc-MIBI scan. Pooled sensitivity and specificity of ${ }^{11} \mathrm{C}$ MET PET in patients with HPT with negative or inconclusive ${ }^{99 \mathrm{~m} T c-M I B I}$ scan were $86 \%$ and $86 \%$, respectively. The authors concluded that ${ }^{11} \mathrm{C}$-MET PET can be a useful functional imaging modality in patients with negative or inconclusive ${ }^{99 \mathrm{~m}}$ Tc-MIBI scan [34].

Caldarella and co-authors investigated the diagnostic performance of ${ }^{11} \mathrm{C}$-MET PET in patients with suspected parathyroid adenoma. Pooled sensitivity and DR values of ${ }^{11} \mathrm{C}$-MET PET in patients with suspected parathyroid adenoma were $81 \%$ (95\%CI: $74-86 \%$ ) and $70 \%$ (95\%CI: 62-77\%), respectively, on a per-patientbased analysis. The authors also concluded that ${ }^{11} \mathrm{C}$-MET PET could be helpful when conventional imaging techniques are negative or inconclusive in localizing parathyroid adenoma [35].

An evidence-based article by Kim and colleagues investigated the diagnostic performance of radiolabelled choline for localization of hyperfunctioning parathyroid gland in patients with HPT. The pooled sensitivity for radiolabelled choline PET/CT was 90\% (95\% CI: 86-94\%) and the pooled specificity $94 \%$ (95\%CI: 90-96\%) [36].

These findings are in line with a recent metaanalysis on the diagnostic performance of radiolabelled choline PET for detecting hyperfunctioning parathyroid glands: on a per-patient analysis, the sensitivity was 95\% (95\%CI: 92-97\%) and the positive predictive value was $97 \%$ (95\% CI: 95-98\%); on a per-lesion analysis, pooled sensitivity and PPV were 92\% (95\%CI: 88-96) and 92\% (95\% CI: 89-95\%), respectively [37].

\section{References}

1. Dinnes J, Bancos I, Ferrante di Ruffano L, Chortis V, Davenport C, Bayliss S, et al. Management of endocrine disease: imaging for the diagnosis of malignancy in incidentally discovered adrenal masses: a systematic review and meta-analysis. Eur J Endocrinol. 2016;175:R51-64. 
2. Kim SJ, Lee SW, Pak K, Kim IJ, Kim K. Diagnostic accuracy of 18F-FDG PET or PET/CT for the characterization of adrenal masses: a systematic review and meta-analysis. Br J Radiol. 2018;91:20170520.

3. Treglia G, Cocciolillo F, de Waure C, Di Nardo F, Gualano MR, Castaldi P, et al. Diagnostic performance of 18F-dihydroxyphenylalanine positron emission tomography in patients with paraganglioma: a meta-analysis. Eur J Nucl Med Mol Imaging. 2012;39:1144-53.

4. Rufini V, Treglia G, Castaldi P, Perotti G, Giordano A. Comparison of metaiodobenzylguanidine scintigraphy with positron emission tomography in the diagnostic work-up of pheochromocytoma and paraganglioma: a systematic review. Q J Nucl Med Mol Imaging. 2013;57:122-33.

5. Kan Y, Zhang S, Wang W, Liu J, Yang J, Wang Z. $68 \mathrm{Ga}$-somatostatin receptor analogs and 18F-FDG PET/CT in the localization of metastatic pheochromocytomas and paragangliomas with germline mutations: a meta-analysis. Acta Radiol. 2018;59:1466-74.

6. Han S, Suh CH, Woo S, Kim YJ, Lee JJ. Performance of $68 \mathrm{Ga}$ DOTA conjugated somatostatin receptortargeting peptide PET in detection of pheochromocytoma and paraganglioma: a systematic review and metaanalysis. J Nucl Med. 2019;60:369-76.

7. Bleeker G, Tytgat GA, Adam JA, Caron HN, Kremer LC, Hooft L, et al. 123I MIBG scintigraphy and 18F-FDG-PET imaging for diagnosing neuroblastoma. Cochrane Database Syst Rev. 2015;9:CD009263.

8. Xia J, Zhang H, Hu Q, Liu SY, Zhang LQ, Zhang A, et al. Comparison of diagnosing and staging accuracy of PET (CT) and MIBG on patients with neuroblastoma: systemic review and meta-analysis. J Huazhong Univ Sci Technolog Med Sci. 2017;37:649-60.

9. Treglia G, Kakhki VR, Giovanella L, Sadeghi R. Diagnostic performance of fluorine-18fluorodeoxyglucose positron emission tomography in patients with Merkel cell carcinoma: a systematic review and meta analysis. Am J Clin Dermatol. 2013; 14:437-47.

10. Singh S, Poon R, Wong R, Metser U. 68Ga PET imaging in patients with neuroendocrine tumors: a systematic review and meta-analysis. Clin Nucl Med. 2018;43:802-10.

11. Barrio M, Czernin J, Fanti S, Ambrosini V, Binse I, Du L, et al. The impact of somatostatin receptordirected PET/CT on the management of patients with neuroendocrine tumor: a systematic review and metaanalysis. J Nucl Med. 2017;58:756-61.

12. Deppen SA, Blume J, Bobbey AJ, Shah C, Graham $\mathrm{MM}$, Lee P, et al. ${ }^{68} \mathrm{Ga}$-DOTATATE compared with ${ }^{111}$ In-DTPA-octreotide and conventional imaging for pulmonary and gastroenteropancreatic neuroendocrine tumors: a systematic review and meta-analysis. J Nucl Med. 2016;57:872-8.

13. Treglia G, Castaldi P, Rindi G, Giordano A, Rufini V. Diagnostic performance of Gallium-68 somatostatin receptor PET and PET/CT in patients with thoracic and gastroenteropancreatic neuroendocrine tumours: a meta-analysis. Endocrine. 2012;42:80-7.

14. Geijer H, Breimer LH. Somatostatin receptor PET/ CT in neuroendocrine tumours: update on systematic review and meta-analysis. Eur J Nucl Med Mol Imaging. 2013;40:1770-80.

15. Yang J, Kan Y, Ge BH, Yuan L, Li C, Zhao W. Diagnostic role of Gallium-68 DOTATOC and Gallium-68 DOTATATE PET in patients with neuroendocrine tumors: a meta-analysis. Acta Radiol. 2014;55:389-98.

16. Blomberg BA, Moghbel MC, Saboury B, Stanley CA, Alavi A. The value of radiologic interventions and (18)F-DOPA PET in diagnosing and localizing focal congenital hyperinsulinism: systematic review and meta-analysis. Mol Imaging Biol. 2013;15:97-105.

17. Yang J, Hao R, Zhu $X$. Diagnostic role of 18F-dihydroxyphenylalanine positron emission tomography in patients with congenital hyperinsulinism: a meta-analysis. Nucl Med Commun. 2013;34:347-53.

18. Treglia G, Mirk P, Giordano A, Rufini V. Diagnostic performance of fluorine-18-dihydroxyphenylalanine positron emission tomography in diagnosing and localizing the focal form of congenital hyperinsulinism: a meta-analysis. Pediatr Radiol. 2012;42:1372-9.

19. Nayan S, Ramakrishna J, Gupta MK. The proportion of malignancy in incidental thyroid lesions on 18-FDG PET study: a systematic review and meta-analysis. Otolaryngol Head Neck Surg. 2014;151:190-200.

20. Qu N, Zhang L, Lu ZW, Wei WJ, Zhang Y, Ji QH. Risk of malignancy in focal thyroid lesions identified by (18)F-fluorodeoxyglucose positron emission tomography or positron emission tomography/computed tomography: evidence from a large series of studies. Tumour Biol. 2014;35(6):6139-47.

21. Treglia G, Bertagna F, Sadeghi R, Verburg FA, Ceriani L, Giovanella L. Focal thyroid incidental uptake detected by ${ }^{18} \mathrm{~F}$-fluorodeoxyglucose positron emission tomography. Meta-analysis on prevalence and malignancy risk. Nuklearmedizin. 2013;52:130-6.

22. Wang N, Zhai H, Lu Y. Is fluorine-18 fluorodeoxyglucose positron emission tomography useful for the thyroid nodules with indeterminate fine needle aspiration biopsy? A meta-analysis of the literature. J Otolaryngol Head Neck Surg. 2013;42:38.

23. Haslerud T, Brauckhoff K, Reisæter L, Küfner Lein R, Heinecke A, Varhaug JE, et al. F18-FDG-PET for recurrent differentiated thyroid cancer: a systematic meta-analysis. Acta Radiol. 2016;57:1193-200.

24. Caetano R, Bastos CR, de Oliveira IA, da Silva RM, Fortes CP, Pepe VL, et al. Accuracy of positron emission tomography and positron emission tomography$\mathrm{CT}$ in the detection of differentiated thyroid cancer recurrence with negative (131) I whole-body scan results: a meta-analysis. Head Neck. 2016;38:316-27.

25. Schütz F, Lautenschläger C, Lorenz K, Haerting J. Positron emission tomography (PET) and PET/ CT in thyroid cancer: a systematic review and metaanalysis. Eur Thyroid J. 2018;7:13-20. 
26. Kim SJ, Lee SW, Pak K, Shim SR. Diagnostic performance of PET in thyroid cancer with elevated anti-Tg Ab. Endocr Relat Cancer. 2018;25:643-52.

27. Santhanam P, Khthir R, Solnes LB, Ladenson $\mathrm{PW}$. The relationship of $\mathrm{BRAF}^{\mathrm{V} 600 \mathrm{E}}$ mutation status to FDG PET/CT avidity in thyroid cancer: a review and meta-analysis. Endocr Pract. 2018;24:21-6.

28. Santhanam P, Taieb D, Solnes L, Marashdeh W, Ladenson PW. Utility of I-124 PET/CT in identifying radioiodine avid lesions in differentiated thyroid cancer: a systematic review and meta-analysis. Clin Endocrinol. 2017;86:645-51.

29. Treglia G, Villani MF, Giordano A, Rufini V. Detection rate of recurrent medullary thyroid carcinoma using fluorine-18 fluorodeoxyglucose positron emission tomography: a meta-analysis. Endocrine. 2012;42:535-45.

30. Cheng X, Bao L, Xu Z, Li D, Wang J, Li Y. ${ }^{18}$ F-FDGPET and ${ }^{18} \mathrm{~F}-\mathrm{FDG}-\mathrm{PET} / \mathrm{CT}$ in the detection of recurrent or metastatic medullary thyroid carcinoma: a systematic review and meta-analysis. J Med Imaging Radiat Oncol. 2012;56:136-42.

31. Treglia G, Cocciolillo F, Di Nardo F, Poscia A, de Waure C, Giordano A, et al. Detection rate of recurrent medullary thyroid carcinoma using fluorine-18 dihydroxyphenylalanine positron emission tomography: a meta-analysis. Acad Radiol. 2012;19(10):1290-9.
32. Treglia G, Tamburello A, Giovanella L. Detection rate of somatostatin receptor PET in patients with recurrent medullary thyroid carcinoma: a systematic review and a meta-analysis. Hormones. 2017;16:362-72.

33. Kluijfhout WP, Pasternak JD, Drake FT, Beninato T, Gosnell JE, Shen WT. Use of PET tracers for parathyroid localization: a systematic review and metaanalysis. Langenbeck's Arch Surg. 2016;401:925-35.

34. Yuan L, Liu J, Kan Y, Yang J, Wang X. The diagnostic value of 11C-methionine PET in hyperparathyroidism with negative 99mTc-MIBI SPECT: a meta-analysis. Acta Radiol. 2017;58:558-64.

35. Caldarella C, Treglia G, Isgrò MA, Giordano A. Diagnostic performance of positron emission tomography using ${ }^{11} \mathrm{C}$-methionine in patients with suspected parathyroid adenoma: a meta-analysis. Endocrine. 2013;43:78-83.

36. Kim SJ, Lee SW, Jeong SY, Pak K, Kim K. Diagnostic performance of F-18 fluorocholine PET/CT for parathyroid localization in hyperparathyroidism: a systematic review and meta-analysis. Horm Cancer. 2018;9:440-7.

37. Treglia G, Piccardo A, Imperiale A, Strobel K, Kaufmann PA, Prior JO, et al. Diagnostic performance of choline PET for detection of hyperfunctioning parathyroid glands in hyperparathyroidism: a systematic review and meta-analysis. Eur J Nucl Med Mol Imaging. 2019;46:751-65.

Open Access This chapter is licensed under the terms of the Creative Commons Attribution 4.0 International License (http://creativecommons.org/licenses/by/4.0/), which permits use, sharing, adaptation, distribution and reproduction in any medium or format, as long as you give appropriate credit to the original author(s) and the source, provide a link to the Creative Commons license and indicate if changes were made.

The images or other third party material in this chapter are included in the chapter's Creative Commons license, unless indicated otherwise in a credit line to the material. If material is not included in the chapter's Creative Commons license and your intended use is not permitted by statutory regulation or exceeds the permitted use, you will need to obtain permission directly from the copyright holder. 